\title{
A simple, ultrahigh vacuum compatible scanning tunneling microscope for use at variable temperatures
}

\author{
F. Mugele, Ch. Kloos, and P. Leiderer \\ Fakultät für Physik, Universität Konstanz, Postfach 5560 M675, 78434 Konstanz, Germany \\ R. Möller \\ Universität Stuttgart, IV. Physikal. Institut, Pfaffenwaldring 57, 70550 Stuttgart, Germany
}

(Received 7 September 1995; accepted for publication 24 March 1996)

\begin{abstract}
We present the construction of a very compact scanning tunneling microscope (STM) which can be operated at temperatures between 4 and $350 \mathrm{~K}$. The tip and a tiny tip holder are the only movable parts, whereas the sample and the piezoscanner are rigidly attached to the body of the STM. This leads to an excellent mechanical stability. The coarse approach system relies on the slip-stick principle and is operated by the same piezotube which is used for scanning. As an example of the performance of the device, images of a $\mathrm{NbSe}_{2}$ surface with atomic resolution are obtained.
\end{abstract}

\section{INTRODUCTION}

Since its invention by Binnig and Rohrer in 1982, ${ }^{1}$ scanning tunneling microscopy (STM) has become a standard technique in surface science. According to the requirements of different experimental conditions, a large number of STM designs have been developed. Application at low temperature usually leads to several difficulties, like limited space, difficult mechanical access, or problems with vibrational isolation in connection with good thermal coupling, etc. Various constructions have been presented to overcome these problems. ${ }^{2-7}$ A common feature for most of them is a coarse approach mechanism based on the slip-stick principle first presented by Pohl. ${ }^{8}$ It provides a simple and reliable possibility to move parts of the STM without need for complicated mechanical constructions used in earlier designs of low-temperature STMs. ${ }^{6}$

The concept discussed in this article represents a simplification for low-temperature STM construction by reducing the number of components and movable parts. This permits a very compact design with external dimensions only slightly larger than the piezoscanner. The small size and simplicity also yields an improved mechanical stability which makes the STM much less susceptible to mechanical vibrations. It has been one goal of the construction presented here that the STM can be operated without vibrational damping, except for some coarse isolation of the cryostat.

\section{DESIGN}

The design of the STM is shown schematically in Fig. 1. It consists of a tube made from invar steel to which the piezotube is attached from one side and the sample from the other side. In detail, the piezotube is glued ${ }^{9}$ to the top plate made from macor which is then screwed onto the invar tube. The bottom plate is also screwed onto the invar tube but with an insulating Teflon ring in between, because we measure the tunneling current at the bottom plate. The bottom plate has a thread, and the sample is mounted on a large screw which can be inserted into this thread. Thus the samples can be exchanged in a very convenient way by just exchanging the large screw which is the sample holder. A carbon resistor mounted on the bottom plate allows for heating the whole microscope to $350 \mathrm{~K}$. The temperature is measured by a $\mathrm{Si}$ diode likewise mounted on the bottom plate, opposite to the heater. Two apertures in the invar tube provide optical access to the sample.

The tip is mounted in a tip holder consisting of two small, ferromagnetic steel balls ( $2 \mathrm{~mm}$ diam) for commercial ball bearings which are connected by a $1 \mathrm{~mm}$ steel tube. The tip is simply inserted into the steel tube and fixed by squeezing the tube. This tip looks like a dumb bell, though weighing less than $0.1 \mathrm{~g}$. The tip holder is forced onto a track by a small magnet positioned inside the invar tube. The track itself consists of two parallel steel rods which are glued to the bottom end of the piezotube via a supporting part made from electrically insulating macor. The tunneling voltage is applied to the tip through the track which is connected electrically by a thin wire. Coarse approach is performed by applying an appropriate wave form to the four outer electrodes of the piezotube. This leads to a motion of the tip holder along the track towards the surface. Scanning is performed by applying usual, computer generated voltage ramps to the $x$ and $y$ electrodes of the same piezotube. The use of only one piezotube for both coarse approach and scanning is the basic idea of this STM construction, which makes it so simple and mechanically stable since the components, except the tip, are fixed together tightly.

For the operation at cryogenic temperatures the STM is mounted in an indium sealed sample cell with two windows, immersed directly into liquid helium. We use an ordinary ${ }^{4} \mathrm{He}$ glass cryostat which is surrounded by a liquid $\mathrm{N}_{2}$ Dewar. When the STM is operated only down to the temperature range of liquid $\mathrm{N}_{2}$, the inner Dewar is filled with $\mathrm{He}$ gas. The whole cryostat is mounted on a heavy marble plate which rests on polymeric vibration damping material. There is no vibration isolation of the STM inside the cryostat.

The standard procedure for a low-temperature experiment consists of heating the whole STM to a temperature of $350 \mathrm{~K}$ and floating the sample cell several times with $\mathrm{He}$ gas. Afterwards we pump for $12 \mathrm{~h}$ with a turbomolecular pump, 


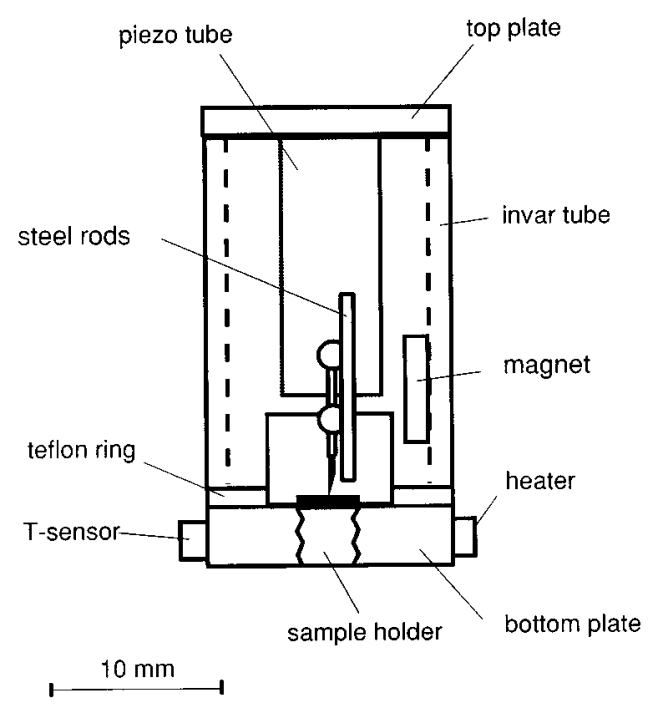

FIG. 1. Cross section of our STM.

keeping the STM at $350 \mathrm{~K}$ all the time. Finally, liquid $\mathrm{N}_{2}$ is introduced into the outer Dewar while the inner one is filled with $\mathrm{He}$ gas for thermal contact. During cooling a temperature difference of about $80 \mathrm{~K}$ is maintained between the STM and the walls of the sample cell. This prevents residual gas from freezing out on the sample. The cooling procedure from 300 to $80 \mathrm{~K}$ takes about $2 \mathrm{~h}$, the following $\mathrm{He}$ transfer about $20 \mathrm{~min}$.

\section{OPERATION}

The most crucial point for a reliable slip-stick motor is the relationship between the static and dynamic friction forces on one hand and the inertial forces of the sliding parts. The condition for the onset of sliding is

$$
a>\left(\mu_{\text {stat }} F_{\text {norm }} / m\right)
$$

where $a$ is the acceleration of the tip holder, $\mu_{\text {stat }}$ is the coefficient of static friction and $F_{\text {norm }}$ the normal force between the track and the tip holder, and $m$ is the mass of the tip holder and the tip. When the slip-stick motor is operated in vertical direction, the gravitational acceleration $g$ has to be added or subtracted on the right-hand side of Eq. (1) when the tip is to move upwards or downwards, respectively. The system switches back from the sliding regime to the riding regime when the relative velocity between the track and the tip holder becomes zero, either because the tip holder has picked up enough momentum by the dynamic frictional forces or because the tracks are decelerated again by the exciting wave form applied to the piezotube. Experimentally, the coefficients of friction are very hard to control, especially because they are subject to large variations upon temperature reduction. Since the mass of the tip holder is fixed, there are essentially two parameters which govern the reliability of the coarse approach system: both the normal force $F_{\text {norm }}$ and the acceleration $a$ were found to be crucial. We obtained reliable performance of different prototypes of this STM for normal forces roughly between 5 and 50 times the gravity of the tip
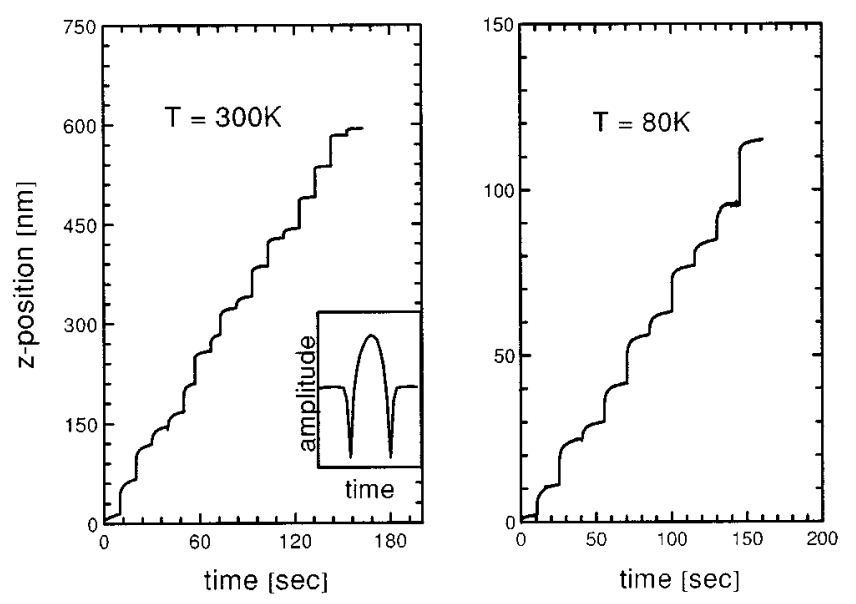

FIG. 2. Two sequences of individual steps of the coarse approach system taken at 300 and $80 \mathrm{~K}$, respectively. The inset shows the cycloidal type wave form used for the excitation.

holder. In order to control $F_{\text {norm }}$ we did not follow the usual concept $^{2-4}$ of using springs and adjusting screws for two reasons: (i) the springs and adjusting screws lead to a more complicated design of the STM and possibly to additional mechanical resonance frequencies ${ }^{2}$ and (ii) $F_{\text {norm }}$ may undergo changes upon temperature variation and it may have to be readjusted frequently. Therefore we chose to create $F_{\text {norm }}$ with a magnet, thereby avoiding these problems. The magnetic field at the position of the sample is on the order of $50 \mathrm{G}$ and thus does not influence STM experiments, unless magnetic properties are to be investigated explicitly. ${ }^{10}$

The acceleration $a$ is determined by the shape and the amplitude of the exciting wave form applied to the piezotube. Like in different previous publications, ${ }^{3,4}$ we found that the appropriate wave form is cycloidal. We generate this cycloidal wave form shown in the inset of Fig. 2 using an analog circuit taken from Blackford et al. ${ }^{4}$ There is a rather broad frequency range from about $500 \mathrm{~Hz}$ to $2 \mathrm{kHz}$ in which successful operation could be obtained for amplitudes exceeding some frequency-dependent threshold voltages. The minimum threshold voltage was $30 \mathrm{~V}$. Both optimum frequency and threshold voltage change upon temperature variation. We typically use $800 \mathrm{~Hz}$ and $120 \mathrm{~V}$ between room temperature and $120 \mathrm{~K}$ and $1.6 \mathrm{kHz}$ and $200 \mathrm{~V}$ at lower temperatures. These parameter values lead to an approach speed of about $0.1 \mathrm{~mm} / \mathrm{s}$. The size of the individual steps was measured by making use of the STM itself. It was determined from the difference in the voltage which had to be applied to the $z$-electrode of the piezo in order to bring the tip into tunneling distance before and after individual coarse approach steps. The individual coarse approach steps were performed by applying a short cycle of the cycloid wave form to the outer piezoelectrodes. Figure 2 shows two typical series of individual steps taken at 300 and $80 \mathrm{~K}$, respectively. The step size calculated from these curves is $(36 \pm 14)$ and $(11 \pm 4) \mathrm{nm}$, respectively. The repoducibility of the step size can be improved by cleaning the tracks with ethanol before every experiment. However, this is not necessary for ordinary, reliable operation. 
In the first experiments we have recorded STM images of $\mathrm{NbSe}_{2}$. The hexagonal atomic lattice of $\mathrm{NbSe}_{2}$ could be resolved at room temperature, $80 \mathrm{~K}$, and $4 \mathrm{~K}$. From the apparent interatomic spacing at the different temperatures we derive a reduction of the piezofactor of our particular piezoelectric ceramic ${ }^{11}$ by a factor of 2.4 and 3.9 with respect to room temperature for 80 and $4 \mathrm{~K}$, respectively.

In conclusion, we have presented the construction of a very compact type of low-temperature STM which requires only one piezoelectric tube. The size of the STM is essentially determined by the dimensions of the piezotube. Neither vibration isolation nor mechanical access are necessary for the operation of the STM. The coarse approach system is based on the slip-stick principle and works reliably between 4 and $350 \mathrm{~K}$, independent of its orientation with respect to gravity. The performance of the STM is demonstrated with atomically resolved images of $\mathrm{NbSe}_{2}$ at different temperatures. Besides, we have been able to demonstrate that this type of STM works reliably in ultrahigh vacuum as well.

\section{ACKNOWLEDGMENTS}

The authors would like to thank J. Boneberg, C. Baur, and A. Rettenberger for fruitful discussions. Financial support by the Deutsche Forschungsgemeinschaft, SFB 306, is gratefully acknowledged.

${ }^{1}$ G. Binnig and H. Rohrer, Helv. Phys. Acta 55, 726 (1982).

${ }^{2}$ I. B. Altfeder and A. P. Volodin, Rev. Sci. Instrum. 64, 3157 (1993).

${ }^{3}$ Ch. Renner, Ph. Niedermann, A. D. Kent, and O. Fischer, Rev. Sci. Instrum. 61, 965 (1990).

${ }^{4}$ B. L. Blackford, M. H. Jericho, and M. G. Boudreau, Rev. Sci. Instrum. 63, 2206 (1992).

${ }^{5}$ S. Kleindiek and K. H. Herrmann, Rev. Sci. Instrum. 64, 692 (1993).

${ }^{6}$ C. A. Lang, M. M. Dovek, and C. F. Quate, Rev. Sci. Instrum. 60, 3109 (1989).

${ }^{7}$ R. Gaisch, J. K. Gimzewski, B. Reihl, R. R. Schlittler, M. Tschudy, and W. D. Schneider, Ultramicroscopy 42, 1621 (1992).

${ }^{8}$ D. W. Pohl, Rev. Sci. Instrum. 58, 54 (1987).

${ }^{9}$ We use Torr Seal from Varian Vacuum Products, a nonconducting two component epoxy resin, which has the disadvantage of becoming conductive after sometime when too high voltages are applied like, for example, between two piezoelectrodes.

${ }^{10}$ For experiments which require high external magnetic fields this scheme for generating $F_{\text {norm }}$ is obviously not suitable.

${ }^{11}$ We used PIK 155 material from PI Ceramic, Gera, Germany. 